\title{
Full NLO QCD corrections to Higgs-pair production in the Standard Model and beyond
}

\author{
Julien Baglio, ${ }^{a, *}$ Francisco Campanario, ${ }^{b, c}$ Seraina Glaus, ${ }^{c, d}$ Milada Margarete \\ Mühlleitner, ${ }^{c}$ Jonathan Ronca $^{b}$ and Michael Spira ${ }^{e}$ \\ ${ }^{a}$ Theoretical Physics Department, CERN, \\ Esplanade des Particules 1, CH-1211 Geneva 23, Switzerland \\ ${ }^{b}$ Theory Division, IFIC, University of Valencia-CSIC, \\ Parque Científico, C/Catedrático José Beltrán, E-46980 Paterna, Spain \\ ${ }^{c}$ Institute for Theoretical Physics, Karlsruhe Institute of Technology, \\ Wolfgang-Gaede-Straße 1, D-76128 Karlsruhe, Germany \\ ${ }^{d}$ Institute for Nuclear Physics, Karlsruhe Institute of Technology, \\ Hermann-von-Helmholtz-Platz 1, D-76344 Karlsruhe, Germany \\ ${ }^{e}$ Laboratory for Particle Physics, Paul Scherrer Institute, \\ Forschungsstrasse 111, CH-5232 Villigen, Switzerland \\ E-mail: julien.baglio@cern.ch, Francisco.Campanario@ific.uv.es, \\ seraina.glaus@kit.edu, milada.muehlleitner@kit.edu, Jonathan.Ronca@uv.es, \\ Michael.Spira@psi.ch
}

Higgs-pair production is one of the targets of the high-luminosity LHC and of future hadron colliders, as it allows for a direct probe of the trilinear Higgs coupling and hence of the mechanism behind electroweak symmetry breaking. This contribution focuses on the impact of the full nextto-leading order QCD corrections to Higgs-pair production via gluon fusion, the main production mechanism at hadron colliders, in the Standard Model and in Two-Higgs-Doublet models. The uncertainties due to the top-mass scale-and-scheme choice will be discussed.

\footnotetext{
*** Particles and Nuclei International Conference - PANIC2021 ***

*** 5 - 10 September, $2021 * * *$

$* * *$ Online $* * *$
}

\footnotetext{
${ }^{*}$ Speaker
} 

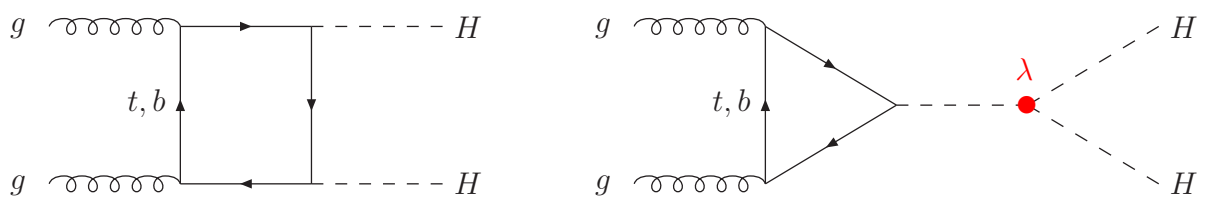

Figure 1: Generic diagrams for $H H$ production at LO in QCD. The $\lambda_{H^{3}}$ contribution is marked in red.

\section{Introduction}

Since the discovery in 2012 of a Higgs boson by ATLAS and CMS collaborations at the CERN Large Hadron Collider (LHC) [1], the detailed study of its properties has not unveiled any deviations from Standard Model (SM) predictions yet [2]. The Higgs self-coupling strengths, however, are crucial properties related to the electroweak symmetry breaking [3] and still un-tested so far. The probe of the triple Higgs coupling $\lambda_{H^{3}}$ is a major goal of the high-luminosity LHC.

Gluon fusion $\mathrm{gg} \rightarrow \mathrm{HH}$ is the dominant production mode at hadron colliders [4] and is already loop-induced at leading order (LO), mediated by heavy-quark loops for which the top-quark contribution is dominant [5]. Higher-order corrections are required to reduce the uncertainties of the theoretical predictions for the cross section and hence of the measurement of $\lambda_{H^{3}}$. The next-to-leading order (NLO) QCD corrections have been first obtained in the heavy top limit (HTL) involving a Born-improvement of the results, leading to a factor-of-two correction [6]. Further refinements have then followed with a large-top-mass expansion [7] as well as with the exact calculation of the real corrections [8]. The full NLO QCD corrections in the SM have now become available thanks to two different numerical calculations, which have found NLO mass effects of order $-15 \%$ on top of the HTL results [9-11]. This result has been supported and confirmed by various analytical approximations [12-15].

In the following we present a very brief overview of the method we have used to obtain the full NLO QCD corrections [10, 11]. Numerical results will be given with emphasis on the topquark scale and scheme uncertainties that is significant, before presenting preliminary results in the Two-Higgs-Doublet-Model (2HDM) of type II which will be published soon.

\section{Overview of the calculation of the NLO QCD corrections}

Gluon fusion production proceeds at LO in QCD via one-loop diagrams with only Yukawa interactions (box diagrams, left-hand side of Figure 1) and with diagrams containing the triple Higgs coupling $\lambda_{H^{3}}$ (triangle diagrams, right-hand side of Figure 1). The virtual corrections for the latter can be taken from known results for off-shell single Higgs production [16] by attaching the Higgs splitting, while the one-particle reducible diagrams can be obtained by recycling known results for $H \rightarrow Z \gamma$ decay, adjusting the kinematics and couplings accordingly. The technically challenging part deals with the two-loop box diagrams. The ultraviolet divergences have been extracted with standard endpoint subtractions, while the infrared divergences have required a suitable local subtraction term. Numerical stability has been achieved thanks to integration by parts of one Feynman parameter after having performed an analytical continuation of the top-quark mass in the complex plane, $m_{t}^{2} \rightarrow m_{t}^{2}(1-i \tilde{\epsilon})$, with $\tilde{\epsilon} \ll 1$. A Richardson extrapolation has been used to reach the narrow-width limit $\tilde{\epsilon} \rightarrow 0$. The strong coupling $\alpha_{s}$ has been renormalised in the $\overline{\mathrm{MS}}$ scheme 

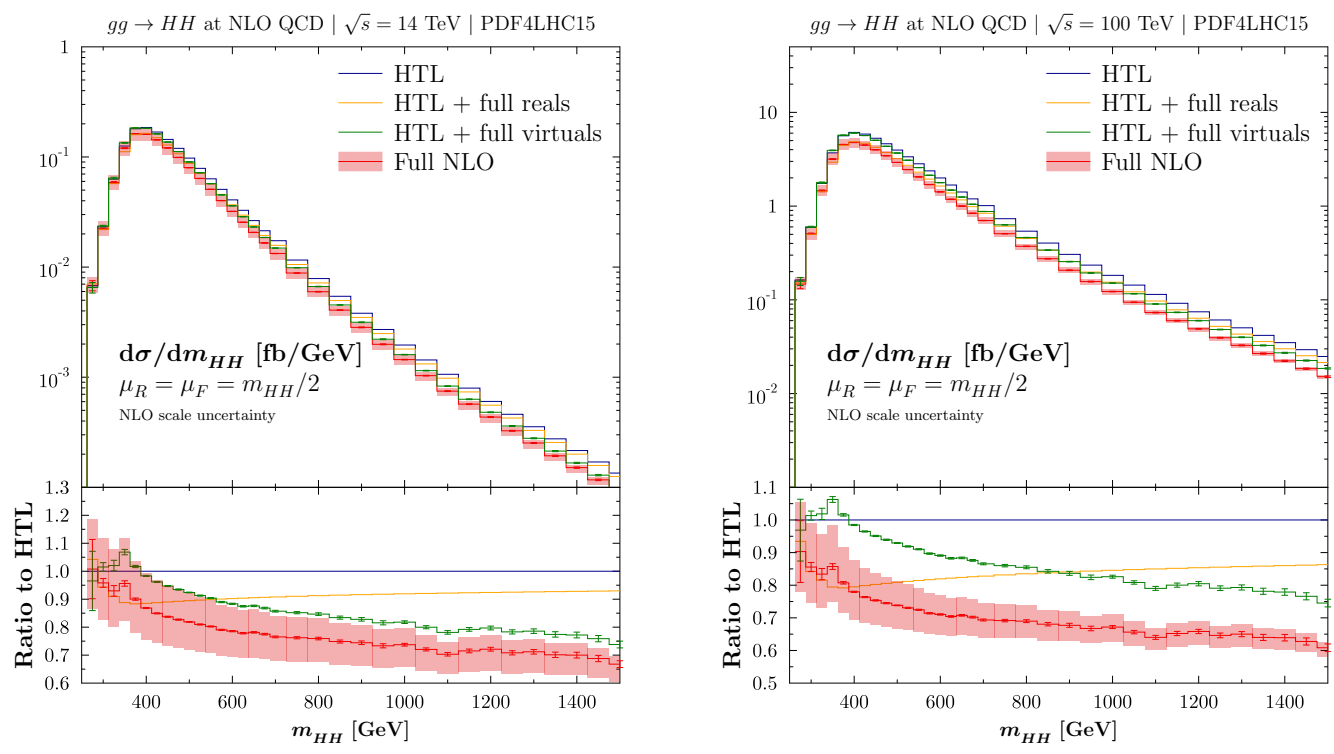

Figure 2: SM Higgs-pair invariant mass distribution at the $14 \mathrm{TeV} \mathrm{LHC} \mathrm{(left)} \mathrm{and} \mathrm{at} \mathrm{a} \mathrm{future} 100 \mathrm{TeV}$ hadron collider (right). The red band represents the scale uncertainty for the full NLO QCD prediction. The lower panels display the ratios of the distribution to the HTL prediction.

with five active flavours and the top-quark mass $m_{t}$ in either the on-shell scheme (OS, our default results) or the $\overline{\mathrm{MS}}$ scheme. The final mass effects of the virtual corrections have been obtained after a numerical integration over the six Feynman parameters and one of the phase-space variables to obtain the invariant Higgs-pair mass $\left(Q=m_{H H}\right)$ distribution. The mass effects in the real corrections have been calculated with FeynArts and FormCalc [17] using the one-loop integral library COLLIER [18]. The HTL contribution has been obtained using HPAIR [6].

\section{SM and 2HDM results}

We present numerical results for the LHC at a centre-of-mass energy of $14 \mathrm{TeV}$ as well as for a planned future circular hadron collider at $100 \mathrm{TeV}$. The OS top-quark mass is fixed to $m_{t}=172.5 \mathrm{GeV}$ and the SM Higgs mass is set to $m_{H}=125 \mathrm{GeV}$. We use the NLO parton distribution functions PDF4LHC15 [19] with LHAPDF6 [20]. For the invariant Higgs-pair distribution we use a grid of $Q$ values from 250 to $1500 \mathrm{GeV}$ and the results are displayed in Figure 2, at $14 \mathrm{TeV}$ (left) and 100 $\mathrm{TeV}$ (right). The full NLO QCD results are in red with a band indicating the renormalisation and factorisation scale uncertainties, around the central value $\mu_{R}=\mu_{F}=Q / 2$. The blue line represents the HTL prediction, the orange line represents the HTL supplemented by the full real corrections, the green line represents the HTL supplemented by the full virtual corrections. It is clear that at large invariant Higgs-pair mass the full NLO QCD corrections are needed, as all blue, orange, green curves fail to even touch the red band. Top-quark mass effects are as large as $-30 \%$ w.r.t. the HTL results. After numerical integration of the differential distribution the total cross section is obtained, $\sigma_{\mathrm{NLO}}^{14} \mathrm{TeV}(g g \rightarrow H H)=32.81(7)_{-12.5 \%}^{+13.5 \%} \mathrm{fb}$, including the usual scale uncertainties.

Our calculation in the $\overline{\mathrm{MS}}$ scheme allows for the first determination ever of the top-quark scale and scheme uncertainties. In each $Q$ bin the maximal and minimal values of the differential cross section define the envelope, when $m_{t}$ is taken either in the OS scheme or in the $\overline{\mathrm{MS}}$ scheme with a 

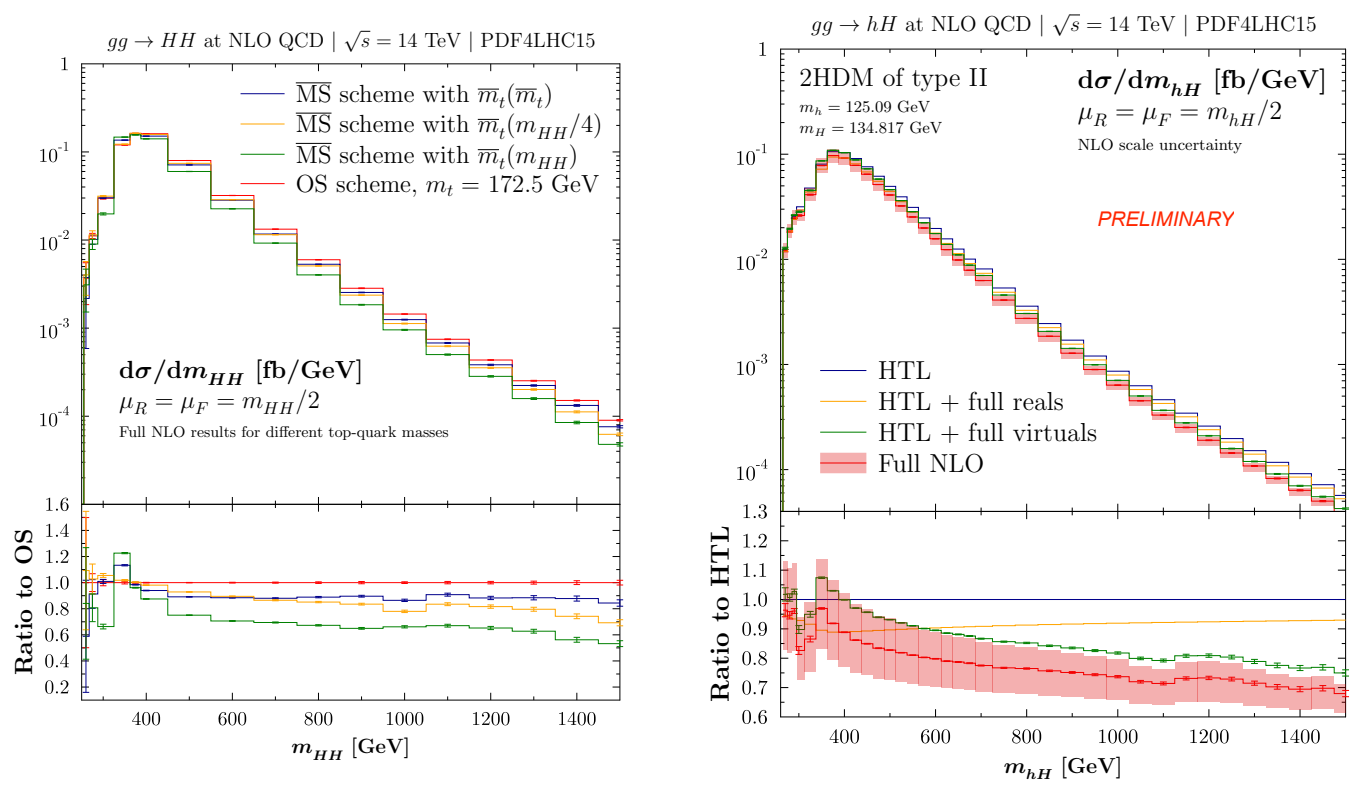

Figure 3: Left: SM Higgs-pair invariant mass distribution at the $14 \mathrm{TeV}$ LHC with different scale and scheme for the top-quark mass. Right: Preliminary results for $h H$ Higgs-pair invariant mass distribution at the $14 \mathrm{TeV}$ LHC in the 2HDM of type II. The lower left panel displays the ratio to the default SM OS prediction, the lower right panel displays the ratio of the distributions to the 2HDM HTL prediction. The red band represents the scale uncertainty for the full NLO QCD prediction

scale $\mu_{t}$ varied between $Q / 4$ and $Q$. The left-hand side of Figure 3 displays the results at the LHC using these different $m_{t}$ scales and schemes. The lower panel shows the ratios of the predictions to the OS scheme. Large effects are found at large $Q$. Integrating the envelope leads to a sizeable $+4 \% /-18 \%$ uncertainty of the total cross section, a factor of two smaller than at LO.

We have also obtained preliminary results in the 2HDM of type II, in order to study the impact of the full NLO QCD corrections in models with an extended Higgs sector. The differential distribution is displayed in Figure 3 (right-hand side) for mixed CP-even Higgs-pair production $h H$ in a benchmark scenario compatible with current experimental results, where the light CP-even Higgs boson is identified with the observed boson, $m_{h}=125.09 \mathrm{GeV}$, while the heavier CP-even Higgs boson has a mass $m_{H}=134.817 \mathrm{GeV}$. We obtain the total cross section $\sigma_{\mathrm{NLO}}^{14} \mathrm{TeV}=18.53(2)_{-12.7 \%}^{+14.0 \%} \mathrm{fb}$, and a differential shape comparable to that of the SM case, with distortions at low $Q$.

\section{Outlook}

The calculation of the full next-to-leading order (NLO) QCD corrections to Higgs pair production cross section at hadron colliders has been presented. The calculation of the two-loop integrals, which contain up to four energy scales, has been performed numerically without any reduction to master integrals. Large top-quark mass effects have been found, of the order of $-15 \%$ for the total cross section. Using both on-shell and $\overline{\mathrm{MS}}$ schemes it has been possible to assess the size of the top-mass scale and scheme uncertainties, of the order of 30\% at large invariant Higgs-pair mass and $+4 \% /-18 \%$ on the total cross section. Combining these findings with the approximated next-to-next-to-leading order (NNLO) prediction [21], the state-of-the-art prediction 
reads $\sigma_{\mathrm{NNLO}}^{14 \mathrm{TeV}}(g g \rightarrow H H)=36.69_{-23 \%}^{+6 \%}$ fb including factorisation scale, renormalisation scale, and top-quark scale and scheme uncertainties.

It will be necessary to determine the full NNLO QCD corrections to decrease the top-mass scale and scheme uncertainties [11], which also arises in other processes such as Higgs+jet production. In the next months a release of the NLO QCD results in the $2 \mathrm{HDM}$ presented here, including also pseudo-scalar pair $A A$ production, is expected.

\section{References}

[1] ATLAS, Phys. Lett. B716 (2012) 1-29; CMS, Phys. Lett. B716 (2012) 30-61.

[2] ATLAS, Phys. Rev. D101 (2020), 012002; CMS, JHEP 01 (2021), 148.

[3] P. W. Higgs, Phys. Lett. 12 (1964), 132-133; F. Englert and R. Brout, Phys. Rev. Lett. 13 (1964), 321-323; P. W. Higgs, Phys. Rev. Lett. 13 (1964), 508-509; G. S. Guralnik, C. R. Hagen and T. W. B. Kibble, Phys. Rev. Lett. 13 (1964), 585-587; P. W. Higgs, Phys. Rev. 145 (1966), 1156-1163; T. W. B. Kibble, Phys. Rev. 155 (1967), 1554-1561.

[4] B. Di Micco et al., Rev. Phys. 5 (2020), 100045.

[5] O.J.P. Eboli, G.C. Marques, S.F. Novaes and A.A. Natale, Phys. Lett. B197 (1987), 269-272; E. W. N. Glover and J. J. van der Bij, Nucl. Phys. B309 (1988), 282-294.

[6] S. Dawson, S. Dittmaier and M. Spira, Phys. Rev. D58 (1998), 115012.

[7] J. Grigo, K. Melnikov and M. Steinhauser, Nucl. Phys. B888 (2014), 17-29; J. Grigo, J. Hoff and M. Steinhauser, Nucl. Phys. B900 (2015), 412-430.

[8] R. Frederix et al., Phys. Lett. B732 (2014), 142-149; F. Maltoni, E. Vryonidou and M. Zaro, JHEP 11 (2014), 079.

[9] S. Borowka et al., Phys. Rev. Lett. 117 (2016), 012001 [erratum: Phys. Rev. Lett. 117 (2016), 079901]; S. Borowka et al., JHEP 10 (2016), 107.

[10] J. Baglio et al., Eur. Phys. J. C79 (2019), 459; J. Baglio et al., JHEP 04 (2020), 181.

[11] J. Baglio et al., Phys. Rev. D103 (2021), 056002.

[12] R. Gröber, A. Maier and T. Rauh, JHEP 03 (2018), 020.

[13] R. Bonciani, G. Degrassi, P. P. Giardino and R. Gröber, Phys. Rev. Lett. 121 (2018), 162003.

[14] J. Davies, G. Mishima, M. Steinhauser and D. Wellmann, JHEP 01 (2019), 176.

[15] G. Wang et al., Phys. Rev. D104 (2021), L051901.

[16] M. Spira, A. Djouadi, D. Graudenz and P. M. Zerwas, Nucl. Phys. B453 (1995), 17-82.

[17] T. Hahn, Comput. Phys. Commun. 140 (2001), 418-431; T. Hahn and M. Perez-Victoria, Comput. Phys. Commun. 118 (1999), 153-165.

[18] A. Denner, S. Dittmaier and L. Hofer, Comput. Phys. Commun. 212 (2017), 220-238.

[19] J. Butterworth et al., J. Phys. G43 (2016), 023001.

[20] A. Buckley et al., Eur. Phys. J. C75 (2015), 132.

[21] M. Grazzini et al., JHEP 05 (2018), 059. 